\title{
Application of platelet-rich plasma accelerates the wound healing process in acute and chronic ulcers through rapid migration and upregulation of cyclin $\mathrm{A}$ and $\mathrm{CDK} 4$ in HaCaT cells
}

\author{
SUNG-AE KIM, HAN-WON RYU, KYU-SUK LEE and JAE-WE CHO \\ Department of Dermatology, Keimyung University School of Medicine, \\ Daegu 700-712, Republic of Korea
}

Received August 2, 2012; Accepted November 29, 2012

DOI: $10.3892 / \mathrm{mmr} .2012 .1230$

\begin{abstract}
Application of autologous platelet-rich plasma (PRP) has been used for chronic wound healing. The aim of this study was to evaluate the effect of PRP on the wound healing processes of both acute and chronic ulcers and the underlying molecular mechanisms involved. We treated 16 patients affected by various acute and chronic ulcers with PRP. We performed molecular studies of cell proliferation, migration assays, immunoblotting and chloramphenicol acetyltransferase (CAT) assays in PRP-treated HaCaT keratinocyte cells. PRP treatment induced increased rates of cell proliferation and cell migration of $\mathrm{HaCaT}$ cells. In addition, the expression of cyclin A and cyclin dependent kinase (CDK) 4 proteins was markedly increased with a low concentration $(0.5 \%)$ of PRP treatment in $\mathrm{HaCaT}$ cells. In 11 patients with chronic ulcers, including stasis ulcers, diabetic ulcers, venous leg ulcers, livedoid vasculitis, claw foot and traumatic ulcers, 9 patients showed 90-100\% epithelization after 15.18 days. In 5 patients with acute ulcers, such as dehiscence, open wound and burn wound, $80-100 \%$ epithelization was achieved between 4 to 20 days. Topical application of PRP to acute and chronic skin ulcers significantly accelerated the epithelization process, likely through upregulation of the cell cycle regulatory proteins cyclin A and CDK4.
\end{abstract}

\section{Introduction}

Platelet-rich plasma (PRP) is a blood-derived fraction containing high concentrations of platelets and growth factors (1). Application of autologous PRP has been reported to facilitate wound healing in several fields, including facilitating bone proliferation in orthopedic surgery, regenerating periodontal ligaments and accelerating the wound healing

Correspondence to: Dr Jae-We Cho, Department of Dermatology, Keimyung University School of Medicine, 194 DongSan-Dong Jung-Gu, Daegu 700-712, Republic of Korea

E-mail: janylove99@dsmc.or.kr

Key words: platelet-rich plasma, skin ulcer, keratinocytes, cell cycles process in diabetic ulcers (2-4). Therefore, in this study, we applied autologous PRP not only to surgical wounds, but also to various forms of ulcers.

Wound healing processes are composed of coagulation, inflammation, migration/proliferation and remodeling phases $(5,6)$. The migration/proliferation of keratinocytes is the key step in accelerating wound healing $(7,8)$. Cell migration/proliferation is precisely regulated by various cell cycle regulatory proteins $(9,10)$. Positive cell cycle regulatory proteins are cyclin D1, cyclin E, cyclin A and their kinase partners, cyclin-dependent protein kinases (CDKs). Among these, cyclin D1-CDK4/6 and cyclin E-CDK2 are the key positive regulatory proteins in the progression of the $\mathrm{G} 1 / \mathrm{S}$ transition phase and cyclin A-CDK2 is important in the G2/M transition phase. Cells in chronic skin ulcers show low cell proliferation rates through the cell cycle and cell cycle arrest, resulting in cellular senescence. Chronic wounds, such as diabetic foot ulcers and venous leg ulcers show impairment of proliferation and migration of keratinocytes into the wound, which result in delayed epithelization $(8,11,12)$. We have previously reported that PRP treatment induced accelerated proliferation and migration of fibroblasts through upregulation of cyclin $\mathrm{E}$ and CDK4, which is important in cell migration and proliferation (13). However, the effect of PRP treatment on migration and proliferation of the HaCaT keratinocyte cell line and its clinical effectiveness in acute and chronic ulcers remain under investigation.

In this study, we investigated the effect of PRP on cell migration, proliferation, and expression of cell cycle regulatory proteins in HaCaT cells and the clinical improvement of PRP-treated ulcers.

\section{Materials and methods}

Materials. Antibodies against cell cycle regulatory proteins (CDK2, CDK4, cyclin A, cyclin D1 and cyclin E) were obtained from Santa Cruz Biotechnology (Santa Cruz, CA, USA). Anti-RB antibody was purchased from Pharmingen (BD Biosciences, San Jose, CA, USA).

Patients. Participants with acute and chronic ulcers were recruited through the dermatology clinic. The recruitment 
A

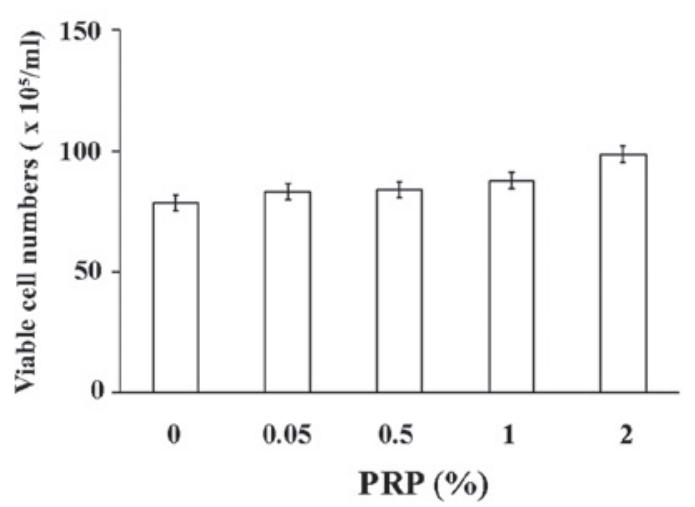



PRP (24h)

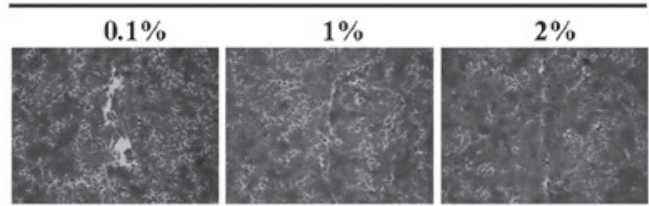

Figure 1. Increased cell proliferation and migration activities of PRP-treated $\mathrm{HaCaT}$ cells. (A) Cells were plated at a density of $15 \times 10^{4} / \mathrm{cm}^{2}$ in 6 -well plates. Cells were cultured with DMEM without serum for 2 days. Medium with various concentrations of PRP was added and cells were cultured for 3 days. Viable cells were counted using trypan blue exclusion assay. (B) Confluent $\mathrm{HaCaT}$ cells were scratched with a plastic micropipette and cells were cultured with DMEM or DMEM with various concentrations of PRP for 1 day. Migration of cells was observed using an inverted microscope. PRP, platelet-rich plasma.

period was between January 2010 and December 2010 and the study was completed in March 2011. Exclusion criteria included the presence of active infection, compromised immune function and coagulation disorders. These processes were approved by the institutional review board of Keimyung University and DongSan Hospital. Written informed patient consent was obtained from the patient.

In total, 16 patients (means \pm SD age, $60 \pm 13,39-83$ years) with various ulcers, including traumatic ulcer, livedoid vasculitis, stasis ulcer, venous leg ulcer, burn ulcer and pressure ulcer, were included. All patients had ulcers involving different body parts: lower leg (7 patients), toe (4), sole (2), heel (1), hand dorsum (1) and finger (1). Ulcer grade was grade 2 (14 patients) or grade 3 ( 2 patients).

PRP preparation. PRP was prepared from the patient's own blood. A small volume of blood $(12 \mathrm{ml})$ was collected using a commercial kit (MyCells Autologous Preparation kit ${ }^{\circledR}$, Holon, Israel) according to the manufacturer's instructions.

PRP application on wound. Activated PRP was applied to the wound as a liquid or gel form according to wound size, location and condition. Prior to the application of PRP, necrotic tissues or eschars were debrided using a scalpel and images were captured with a digital camera (450D; Canon, Tokyo, Japan). Following PRP application, a foam dressing (Allevin ${ }^{\circledR}$, Smith and Nephew, Huntingdon, UK) was placed on the ulcer for $48 \mathrm{~h}$. Time intervals between treatments varied from twice a week to once a week. The number of treatments differed depending on the wound state. After each treatment, the same dermatologist assessed clinical improvement and epithelization rate. Overall degree of ulcer improvement was assessed every week until complete epithelization occurred.

Cell cultures. HaCaT keratinocyte cell lines were maintained at $37^{\circ} \mathrm{C}$ in a humidified atmosphere of $95 \%$ air and $5 \% \mathrm{CO}_{2}$ in DMEM supplemented with $10 \%$ heat inactivated fetal bovine serum, $2 \mathrm{mM}$ glutamine, $100 \mathrm{U} / \mathrm{ml}$ penicillin and $100 \mathrm{~g} / \mathrm{ml}$ strep- tomycin. For the experiments, cells $\left(5 \times 10^{4}\right.$ cells $\left./ \mathrm{ml}\right)$ were seeded in a culture dish and maintained in a tissue culture incubator.

Cell proliferation assay. HaCaT cells were seeded at a density of $15 \times 10^{4}$ cells/well in 6 -well culture plates. The cells were cultured using serum-free DMEM supplemented with 0 (control), $0.05,0.5,1$ or $2 \%$ activated PRP for 3 days. The cultured cells were assayed for proliferation using the trypan blue exclusion method. Briefly, cells were washed with phosphate-buffered saline and stained with trypan blue dye. Viable cells were observed as light reflecting cells, but dead cells were observed as dark cells. The numbers of viable cells were measured under an inverted phase contrast microscope.

Migration assay. For the measurement of cell migration, confluent keratinocytes, which were kept in serum-free medium for $24 \mathrm{~h}$, were wounded with a plastic micropipette tip. After washing, the medium was replaced by serum-free medium or serum-free medium supplemented with $0.01,0.05$, $0.5,1$ or $2 \%$ activated PRP for $24 \mathrm{~h}$. Images of the wounded area were taken every $24 \mathrm{~h}$ by phase-contrast microscopy under crystal violet staining.

Western blot analysis. Whole cell extracts were prepared in lysis buffer [10 mM Tris (pH 7.4), 5 mM EDTA, $130 \mathrm{mM}$ $\mathrm{NaCl}, 1 \%$ Triton $\mathrm{X}-100$, phenylmethylsulphonyl fluoride (PMSF, $10 \mathrm{~g} / \mathrm{ml})$, aprotinin $(10 \mathrm{~g} / \mathrm{ml})$, leupeptin $(10 \mathrm{~g} / \mathrm{ml})$, $5 \mathrm{mM}$ phenanthroline and $28 \mathrm{mM}$ benzamidine- $\mathrm{HCl}]$. The protein concentration of extracts was estimated with Bradford reagent (Bio-Rad Laboratories, Hercules, CA, USA) using bovine serum albumin as the standard. Equal amounts of protein (40 $\mu \mathrm{g} /$ lane) were resolved by $6.5-12 \%$ sodium dodecyl sulfate-polyacrylamide gel electrophoresis and transferred onto a nitrocellulose membrane. The membrane was then washed with Tris-buffered saline $(10 \mathrm{mM}$ Tris, $150 \mathrm{mM} \mathrm{NaCl})$ containing $0.05 \%$ Tween-20 (TBST) and blocked in TBST containing 5\% non-fat dried milk. The membrane was further incubated with respective specific antibodies. The membrane was continuously incubated with appropriate secondary anti- 
Table I. List of PRP-treated acute and chronic patients.

\begin{tabular}{|c|c|c|c|c|c|c|}
\hline No. & Age/gender & Diagnosis & $\begin{array}{c}\text { Duration } \\
\text { of disease (months) }\end{array}$ & Site & $\begin{array}{l}\text { Ulcer } \\
\text { stage }\end{array}$ & $\begin{array}{l}\text { Days of epithelialization } \\
\text { (completeness, \%) }\end{array}$ \\
\hline 1 & $55 / \mathrm{F}$ & Stasis ulcer & 36 & Lt lower leg & II & $24(90)$ \\
\hline 2 & $62 / \mathrm{M}$ & Stasis ulcer & 2 & Lt ant, shin & II & $24(90)$ \\
\hline 3 & $63 / \mathrm{M}$ & DM foot ulcer & 12 & Lt toe & II & $5(60)$ \\
\hline 4 & $51 / \mathrm{M}$ & DM foot ulcer & 18 & Lt sole & III & $20(95)$ \\
\hline 5 & $47 / F$ & DM foot ulcer & 2 & Lt heel & II & $22(90)$ \\
\hline 6 & $63 / \mathrm{M}$ & DM foot ulcer & 4 & Rt 1 st toe tip & II & $6(90)$ \\
\hline 7 & $63 / \mathrm{F}$ & Venous leg ulcer & 7 & Lt med malleolus & II & $20(95)$ \\
\hline 8 & $69 / \mathrm{F}$ & Traumatic ulcer & 5 & Rt lower leg & II & $20(70)$ \\
\hline 9 & $66 / \mathrm{F}$ & Traumatic ulcer & 2 & Rt thumb & II & $7(90)$ \\
\hline 10 & 39/M & Livedoid vasculitis & 2 & Lt med malleolus & II & $21(100)$ \\
\hline 11 & $83 / \mathrm{M}$ & Claw's foot & 4 & MIP joint, 3rd toe & II & $23(95)$ \\
\hline 12 & $69 / \mathrm{M}$ & Dehiscence & 1 & Rt sole & III & $4(80)$ \\
\hline 13 & $60 / \mathrm{M}$ & Open wound & 2 & Rt ant shin & II & $17(85)$ \\
\hline 14 & $44 / \mathrm{M}$ & Pressure ulcer & 2 & Lt lat malleolus & II & $35(100)$ \\
\hline 15 & $73 / \mathrm{M}$ & Burn & 2 & Hand & II & $10(90)$ \\
\hline 16 & $54 / \mathrm{M}$ & Burn & 3 & Lt toe and heel & II & $30(100)$ \\
\hline
\end{tabular}

F, female; M, male; Lt, left; Rt, right; med, medial; lat, lateral; PRP, platelet-rich plasma; DM, diabetes mellitus; MIP, metacarpointerphalangeal joint; ant, anterior.

bodies coupled to horseradish peroxidase and developed in the ECL Western detection reagents (Amersham Pharmacia Biotech, Piscataway, NJ, USA).

\section{Results}

Effect of PRP on cell migration/proliferation rates of the HaCaT keratinocyte cell line. We analyzed the effect of PRP on migration and proliferation rates of the HaCaT keratinocyte cell line. As shown in Fig. 1A, PRP treatment resulted in a dose-dependent increase of the proliferation rates of $\mathrm{HaCaT}$ cells. In addition, PRP treatment resulted in markedly increased rates of migration of $\mathrm{HaCaT}$ cells, even with a low concentration of PRP (0.005-0.5\%; Fig. 1B).

Expression of cell cycle regulatory proteins in the $\mathrm{HaCaT}$ keratinocyte cell line by PRP treatment. Cell cycle regulatory proteins are important in proliferation and migration of $\mathrm{HaCaT}$ keratinocyte cell lines. We have investigated the question of whether PRP treatment promotes the proliferative and migratory activities of $\mathrm{HaCaT}$ cells through upregulation of G1/S or $\mathrm{G} 2 / \mathrm{M}$ transition regulatory proteins. In order to determine the basal expression levels of cell cycle regulatory proteins in $\mathrm{HaCaT}$ cells, cells were cultured without fetal bovine serum (FBS) for 3 days. After serum starvation for 3 days, cells were cultured under 5\% FBS or 2\% (v/v) PRP with DMEM (Fig. 2). Expression levels of G1/S transition regulatory proteins, namely, ppRb, CDK4 and cyclin A, were decreased in serum-starved $\mathrm{HaCaT}$ cells. Notably, 2\% PRP induced increased expression of cyclin A and CDK4 in $\mathrm{HaCaT}$ cells, resulting in increased expression of ppRb. Markedly increased expression of cyclin D1, cyclin E and CDK4 was not observed.



Figure 2. Expression of cell cycle regulatory proteins in PRP-treated HaCaT cells. Cells were cultured without serum for 3 days, followed by the addition of 5\% FBS or 2\% PRP to culture media. Proteins were extracted from cells after $24 \mathrm{~h}$; protein extracts were then subjected to western blot analysis using specific primary antibodies. Similar results were obtained in 2 different experiments. PRP, platelet-rich plasma; FBS, fetal bovine serum; CDK, cyclin-dependent kinase.

Wound healing effect of PRP on acute and chronic skin ulcers. Based on the laboratory data that support the enhancing effect of PRP on wound healing, we performed a clinical application of PRP to acute and chronic wounds and evaluated its clinical wound healing effect. The clinical characteristics and outcomes of patients are summarized in Table I.

Eleven patients with chronic ulcers (cases 1-11) presented with stasis ulcer, diabetic ulcer, venous leg ulcer, livedoid vasculitis, claw foot and traumatic ulcer. The mean period 
A

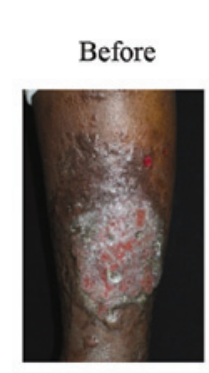

C

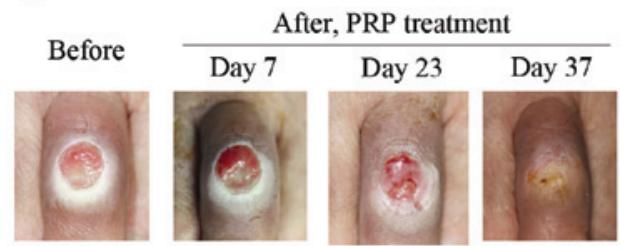

E

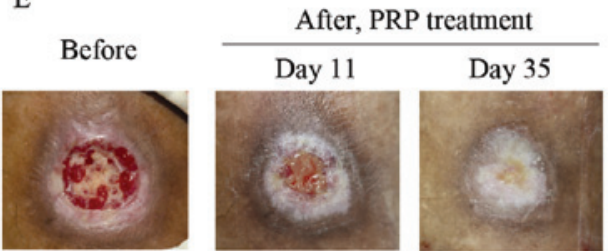

B

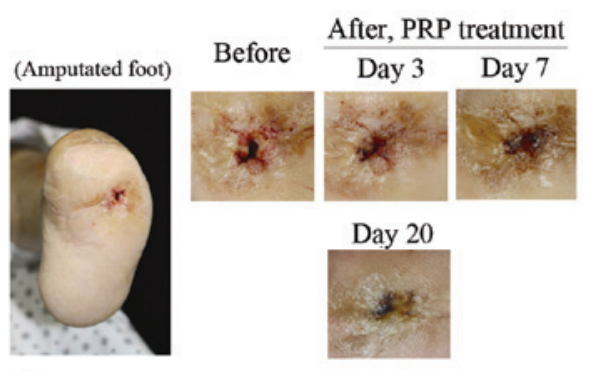

D



After, PRP treatment

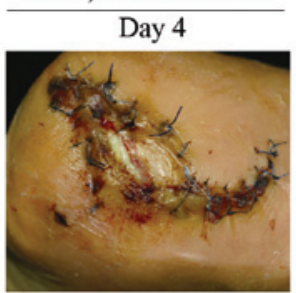

F

Before

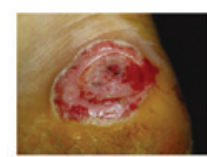

After, PRP treatment



Figure 3. Clinical improvement of various skin ulcers following PRP treatment. PRP was applied onto the following skin ulcers: (A) huge stasis ulcer, (B) non-adhesion wound on an amputated foot, (C) iatrogenic ulcer on claw foot, (D) acute dehiscence wound following surgery, (E) pressure ulcer on lateral malleolus and (F) burn ulcer. PRP gel solution was applied on the ulcer 2 times a week, 2-5 times for each case depending on the clinical status. After PRP treatment, the wounds were covered by a foam dressing for 2 days. PRP, platelet-rich plasma.

of the disease was 8.54 months. However, in 15.18 days, 9 patients showed $90-100 \%$ epithelization. Case 2 presented with large-sized stasis ulcers and had been refractory to standard treatment for 2 months. However, almost a complete epithelization was shown in 24 days after 3 PRP treatments at 3-day intervals (Fig. 3A). In case 4, the patient had been suffering from a penetrating non-adhesion deep ulcer on an amputated foot for 18 months (Fig. 3B). The wound had not been healed by sutures. Thus, we applied PRP gel (6 times) on the ulcer and observed a complete epithelization 20 days after treatment. In case 11, a round-shaped ulcer on the middle interphalangeal joint of the left 3rd toe following corn treatment using cryotherapy (Fig. 3C) also showed epithelization in 23 days after treatment 3 times with PRP gel solution.

In 5 patients with acute ulcers (cases 12-16), an 80-100\% epithelization rate was achieved in 20 days. These wounds had occurred for an average of 2 months, including special indications such as dehiscence (case 12, Fig. 3D), open wound, pressure ulcer (case 14, Fig. 3E) and burn wound (case 16, Fig. 3F). Three months after the treatment, the majority of the patients (90\%), including acute and chronic patients, evaluated the ulcer appearance as having good to excellent improvement and the remaining patients $(10 \%)$ rated the improvement as moderate.

\section{Discussion}

Platelet-rich plasma (PRP) is an autologous preparation of platelets concentrated in plasma. PRP contains $>30$ bioac- tive proteins including PDGF, TGF- $\beta$, VEGF, EGF and bFGF, which have fundamental roles in wound healing (14). These factors are known to regulate processes such as cell migration, attachment, proliferation and differentiation, and promote extracellular matrix (ECM) accumulation by binding to specific cell surface receptors (15). Thus, autologous PRP therapy may be a promising agent for acceleration of the wound healing process through high concentrations of growth factors.

Keratinocytes are important in epithelization during the wound healing process $(8,16)$. In a number of studies, critical defects were found in epithelial cell migration in chronic skin ulcers. Promoting epithelial cell migration enhances wound healing not only in chronic skin ulcers but also in acute ulcers. In this study, we attempted to investigate the effect of PRP on keratinocyte activation, migration, proliferation and expression of cell cycle regulatory proteins. Our data clearly indicated that even an extremely low concentration $(0.5 \%)$ of PRP accelerated the migration of $\mathrm{HaCaT}$ cells. In addition, cell proliferation rates of PRP-treated $\mathrm{HaCaT}$ cells were increased by the upregulation of CDK4 and cyclin A expression. Thus, PRP-induced keratinocyte migration and cell proliferation contribute to the rapid wound healing process in chronic and acute wounds. In our previous study, we identified PRP-induced cell cycle promotion in human skin fibroblasts, which is indicative of the wound healing effect of chronic ulcers (13). Thus, PRP exerts cell cycle progression in keratinocytes and fibroblasts. 
In this study, we investigated the effect of PRP on keratinocyte migration and proliferation, key steps for wound healing in vitro. It is important to note that its clinical effects are not only on chronic ulcers, but also apply to various ulcers such as venous leg ulcer, stasis dermatitis, burn ulcer and acute traumatic ulcer. Clear effects of PRP enhancing re-epithelization of chronic and acute wounds have also been revealed. In the patients, periods of epithelization were shortened from the expected time through enhanced migration and proliferation of keratinocytes by PRP.

We have demonstrated that PRP treatment induced increased rates of cell proliferation and cell migration of HaCaT cells, and showed 90-100\% epithelization in 15.18 days in chronic ulcers, and 80-100\% epithelization was achieved between 4 to 20 days in acute ulcers. Promotion of wound healing in the skin by shortening the epithelization process occurs through upregulation of cyclin A and CDK4 expression in cells, thereby promoting proliferation and migration of keratinocytes.

\section{Acknowledgements}

This study was supported by the Research Promoting Grant from the Keimyung University Dongsan Medical Center in 2011.

\section{References}

1. Arora NS, Ramanayake T, Ren YF and Romanos GE: Plateletrich plasma: a literature review. Implant Dent 18: 303-310, 2009.

2. Azzena B, Mazzoleni F, Abatangelo G, Zavan B and Vindigni V: Autologous platelet-rich plasma as an adipocyte in vivo delivery system: case report. Aesthetic Plast Surg 32: 155-158, 2008.
3. Klaassen MA and Pietrzak WS: Platelet-rich plasma application and heterotopic bone formation following total hip arthroplasty. J Invest Surg 24: 257-261, 2011.

4. Akingboye AA, Giddins S, Gamston P, Tucker A, Navsaria H and Kyriakides C: Application of autologous derived-platelet rich plasma gel in the treatment of chronic wound ulcer: diabetic foot ulcer. J Extra Corpor Technol 42: 20-29, 2010.

5. Behm B, Babilas P, Landthaler M and Schreml S: Cytokines, chemokines and growth factors in wound healing. J Eur Acad Dermatol Venereol 26: 812-820, 2011.

6. Barrientos S, Stojadinovic O, Golinko MS, Brem H and TomicCanic M: Growth factors and cytokines in wound healing. Wound Repair Regen 16: 585-601, 2008.

7. Yang X, Teng Y, Hou N, Fan X, Cheng X, Li J, Wang L, Wang Y, Wu X and Yang X: Delayed re-epithelialization in Ppmla genedeficient mice is mediated by enhanced activation of Smad2. J Biol Chem 286: 42267-42273, 2011.

8. Raja, Sivamani K, Garcia MS and Isseroff RR: Wound re-epithelialization: modulating keratinocyte migration in wound healing. Front Biosci 12: 2849-2868, 2007.

9. Neganova I and Lako M: G1 to S phase cell cycle transition in somatic and embryonic stem cells. J Anat 213: 30-44, 2008.

10. Donjerkovic D and Scott DW: Regulation of the G1 phase of the mammalian cell cycle. Cell Res 10: 1-16, 2000.

11. Henderson EA: The potential effect of fibroblast senescence on wound healing and the chronic wound environment. J Wound Care 15: 315-318, 2006.

12. Suter MM, Schulze K, Bergman W, Welle M, Roosje P and Muller EJ: The keratinocyte in epidermal renewal and defence. Vet Dermatol 20: 515-532, 2009.

13. Cho JW, Kim SA and Lee KS: Platelet-rich plasma induces increased expression of G1 cell cycle regulators, type I collagen, and matrix metalloproteinase-1 in human skin fibroblasts. Int J Mol Med 29: 32-36, 2012.

14. Prakash S and Thakur A: Platelet concentrates: past, present and future. J Maxillofac Oral Sur 10: 45-49, 2011.

15. Ueda $M$ and Nishino $Y$ : Cell-based cytokine therapy for skin rejuvenation. J Craniofac Surg 21: 1861-1866, 2010.

16. Pullar CE and Isseroff RR: Cyclic AMP mediates keratinocyte directional migration in an electric field. J Cell Sci 118 2023-2034, 2005. 